\title{
Gorham-Stout Disease in a Middle-Aged Patient Treated by Posterior Lateral Fusion: A Case Report and Literature Review
}

\author{
Gi Wuk Jang ${ }^{1}$, Sung Hyun Noh ${ }^{2}$ \\ ${ }^{1}$ Department of Neurosurgery, National Health Insurance Service Ilsan Hospital, Goyang, Republic of Korea \\ ${ }^{2}$ Department of Neurosurgery, Ajou University Hospital, Ajou University College of Medicine, Suwon, Republic of Korea
}

Corresponding author:

Sung Hyun Noh

Department of Neurosurgery, Ajou

University Hospital, Ajou University

College of Medicine, 164, World

Cup-ro, Yeongtong-gu, Suwon

16499, Republic of Korea

Tel: +82-31-219-4821

Fax: +82-31-219-4822

E-mail: juwuman11@nate.com

Received: June 2, 2021

Revised: July 8, 2021

Accepted: July 28, 2021
Gorham-Stout disease (GSD) is a rare progressive osteolytic disease with intraosseous lymphangiomatosis. A previously healthy 55-year-old woman presented with left leg hypoesthesia and upper back pain. $k$ pain, back pain, and bilateral shoulder pain. In radiologic examination, there was an osteolytic lesion in T3, 4, 5, 6 vertebra. And a dislocation in the T3/4 vertebrae and a compression fracture in the T4 vertebra were founded. She was treated with posterolateral fusion (PLF) from C7 to 77 . For 1 year, she had no specific symptoms but a new osteolytic lesion was found on thoracic T8 on 1 year follow-up magnetic resonance imaging. Radiotherapy was performed with $40 \mathrm{~Gy}$, and chemotherapy was performed with denosumab. This report described a case of GSD that involved the thoracic spine and was appropriately managed. In the present case of a middle-aged Asian woman, correction of severe thoracic instability and prevention of neurologic compromise were accomplished through PLF from C7 to T7. Recurrence was observed 1 year later, but it was appropriately treated with neoadjuvant therapy.

Key Words: Osteolysis, essential; Spine; Thoracic vertebrae

\section{INTRODUCTION}

Gorham-Stout disease (GSD), also known as "vanishing bone disease" or "massive osteolysis", is a rare disease of unknown etiology that is characterized by progressive osteolysis and absorption with mono- or poly-osteolytic lesions ${ }^{7}$. Gorham disease was first described in 1838; since then, more than 300 cases have been reported, with approximately 60 cases related to the spine. One common complication of the disease is the development of chylothorax, which occurs in approximately $20 \%$ of patients and results in a high mortality rate $^{13)}$. No standard diagnostic tool or therapy exists for the disease so that it can be easily misdiagnosed. Depending on disease severity and extension of organ involvement, commonly used treatments include surgery, radiotherapy, and pharmaceuticals. GSD can be diagnosed at any age, but it is most commonly observed in children and young adults (the average age of diagnosis is 25 years). This study report management of a middle-aged patient with GSD on thoracic spine combined thoracic vertebral dislocation.

\section{CASE REPORT}

A previously healthy 55-year-old Asian woman presented with left leg hypoesthesia and upper back pain. Before being admitted to the hospital, she visited a local clinic and was diagnosed with fibromyalgia with neck pain, back pain, and bilateral shoulder pain. She had a history of a fall 2 weeks prior to admission, after which symptoms were aggravated. One day before visiting our institution, she noticed a change in her gait and hypoesthesia of her left leg. She had no family history of hereditary disease. On physical examination, she exhibited tenderness in the upper back and a slight loss of left leg sensation (about 60\% remained). Thoracic spine computed tomography (CT) revealed a dislocation in the T3/4 vertebrae and a compression fracture in the T4 vertebra. Magnetic resonance imaging (MRI) scans showed a combined pathological fracture of T4 with anterior subluxation of T3 over T4, causing compressive myelopathy/cord contusion. In addition, the T1 to T6 posterior elements and heads of the left $3^{\text {rd }}$ to $5^{\text {th }}$ ribs showed a hypoplastic appearance with or without osteolysis. A whole-body bone scan demonstrated focal uptake at the T4 area due to the known compression fracture and mild uptake at the spinous process area of T6 due to another fracture (Fig. 1). Blood analyses, including multiple myelopathy tests, indicated normal biochemical levels. She was admitted through the emergency room with T3/4 dislocation and cord compression.

In the present case, cord compression and vertebral dislocation required surgical treatment, and the plan was posterolateral fusion (PLF) from C7 to T7. With the patient under general anesthesia, the surgery was performed in a patient in the prone position of Jackson table. After confirming the surgical site on the radiograph, a dorsal midline incision was made. The subcutaneous fat layer and fascia were dissected and the muscles were separated from the vertebral arch plate. T3 and T4 total laminectomy were performed for decompression of the spinal cord. The bones were very loose 

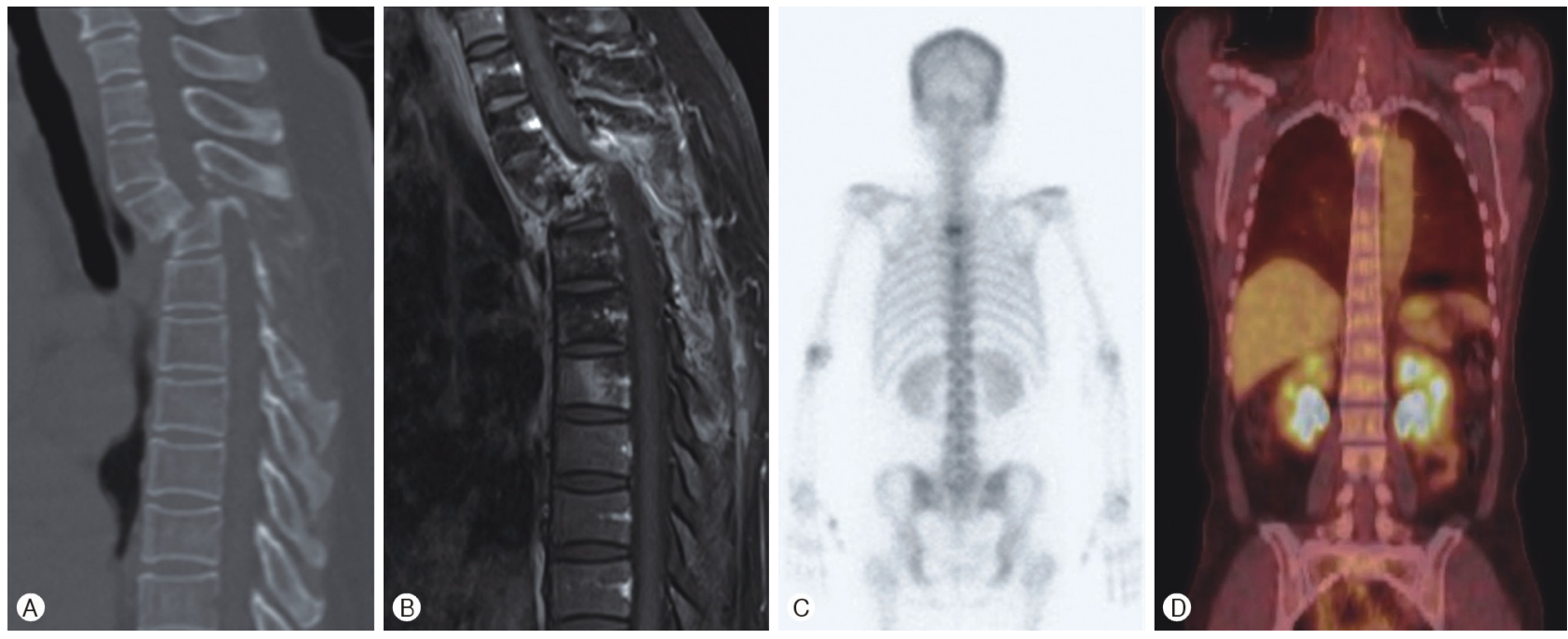

Fig. 1. (A) Preoperative thoracic spine computed tomography imaging shows a dislocation at T3 and T4 level. (B) Preoperative thoracic spine magnetic resonance imaging T1 contrast enhance sagittal image shows a combined pathological fracture of T4 with anterior subluxation of T3 over T4, causing compressive cord contusion. (C) Preoperative whole-body bone scan shows focal uptake appeared at T4 area. (D) Preoperative positron emission tomography shows irregular osteolytic and hypoplastic change at T3, 4, 5, and 6 area.
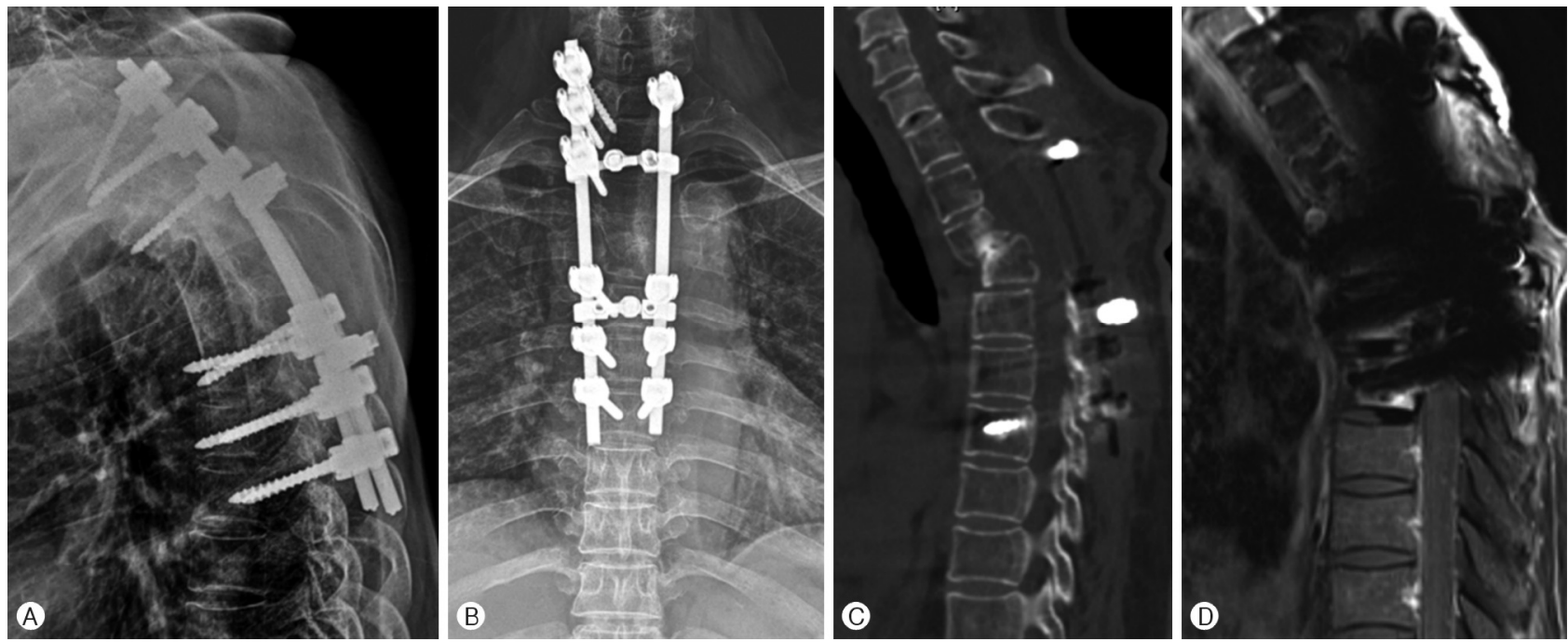

Fig. 2. (A, B) Postoperative lateral and anteroposterior radiograph of the thoracic spine at the follow-up of 4 months after surgery, showing a posterolateral fusion on C7-T7. (C) Computed tomography of the thoracic spine at the follow-up of 4 months after surgery, showing a stabilization of C3/4 vertebral body. (D) Magnetic resonance imaging of the thoracic spine showing a cord decompression at the follow-up of 4 months after surgery.

and, unlike normal bones, they were very thin. Because the bone was very weak and reduction was needed for correcting T3/4 dislocation, pedicle screw insertion was performed up to C7-T7 and bone fusion was conducted with allobone materials. A hemovac drain was placed on the surgical site and the wound was closed along the edge. The surgery was completed uneventfully.

After the operation, her symptoms improved. A Minerva jacket was prescribed to prevent kyphotic deformity and to support the PLF. Postoperative pathological examination of the bone tissue (T3 and 4 vertebral bodies) biopsy revealed GSD with thin-walled vascular proliferation replacing bone marrow and lamellated trabecular bone with fibrous stroma. Two weeks after surgery, chest CT showed bilateral pleural effusions, and the chest was drained on both sides. The drain catheter inserted for the pleural effusions showed that the effusions were exudate, not chylothorax. After 2 weeks, both pleural effusions improved and the drain was removed. Since there is no gold standard for GSD, in this patient, the active phase passed by over 50 years of age, and since there was no other special disease 

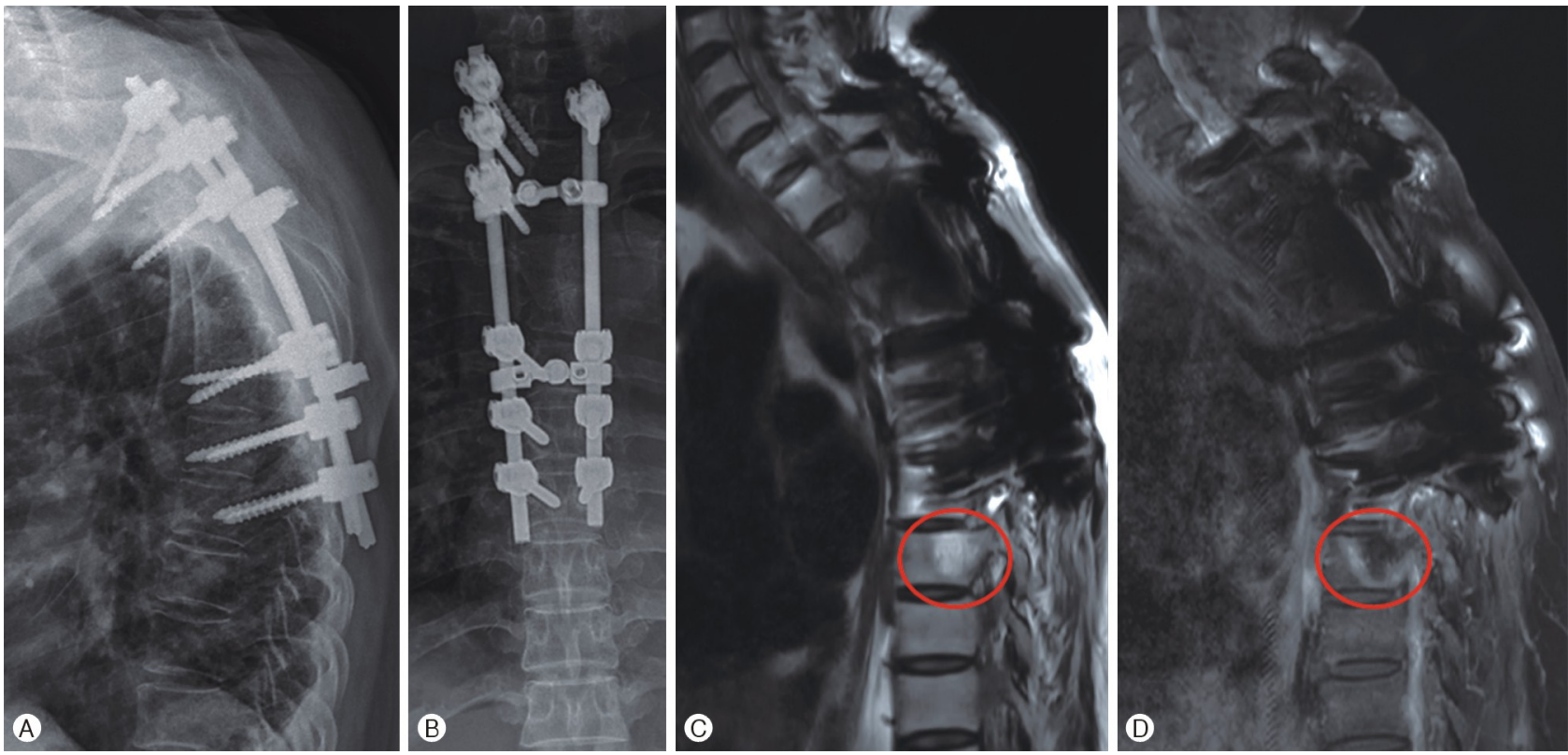

Fig. 3. (A, B) Postoperative lateral and anteroposterior radiograph of the thoracic spine at the follow-up of 1 year after surgery, showing a posterolateral fusion on C7-T7. (C, D) Magnetic resonance imaging of the thoracic spine showing a new osteolytic lesion on T8 left vertebral body, left pedicle on both T2 weighted image and T1 enhance image at the follow-up of lyear after surgery.

before, chemotherapy and radiotherapy were not performed after surgery, and it was decided to follow up. The 4 months after surgery, thoracic spine X-ray and CT scan showed successful reduction and stabilization of the thoracic spine deformity (Fig. 2). A new osteolytic lesion was found involving T8 left vertebral body, left pedicle, bilateral transverse process, left $8^{\text {th }}$ and $9^{\text {th }}$ rib head on 1 year follow up MRI (Fig. 3). Radiotherapy was performed with $40 \mathrm{~Gy}$ with denosumab. There is no recurrence during 8 months after the radiotherapy (Fig. 4).

\section{DISCUSSION}

GSD is also known as vanishing bone disease, massive osteolysis, and disappearing bone disease. Gorham et al. ${ }^{3)}$ reported 24 cases and defined the disease as a syndrome in 1954. Athough the exact pathophysiology of GSD is not known, the correlation between lymphatic proliferation and gradual osteolysis is well-established. Gorham and Stout ${ }^{2)}$ originally posited angiomatosis caused by hyperemia, interrupted bone metabolism, and altered balance to favor osteoclastic activity. GSD presents no hereditary or sex tendency and can occur at any age, although it is more common in adolescents and young adults ${ }^{6}$. As in our case, it is rarely found in people over 50. GSD can involve any part of any bone; however, the skull, shoulder, cenvical vertebrae and pelvis are the most frequently involved sites ${ }^{10)}$. Especially, spinal involvement can lead to instability and neurologic impairment. In our case, GSD occurred in the thoracic spine, and neurologic abnormality occurred due to dislocation. Treatment of spinal GSD requires close observation and multidisciplinary clinical decision-making. Because no standard therapy exists, care of patients with GSD is case specific. Some available

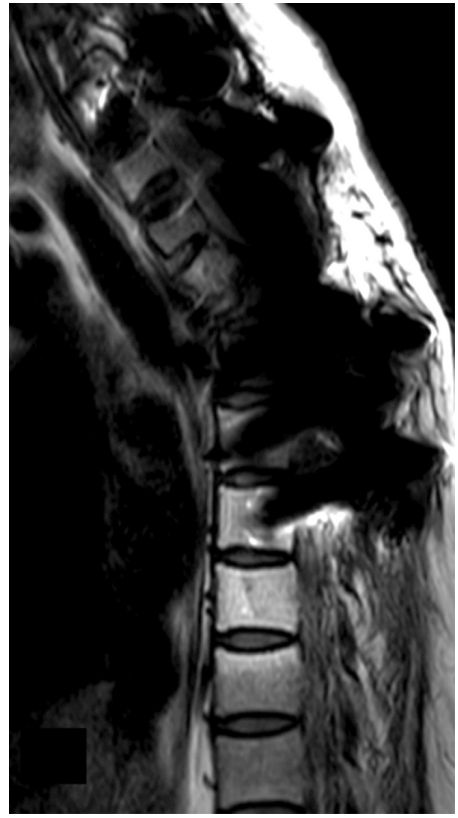

Fig. 4. T2 magnetic resonance imaging thoracic spine at the follow-up after 8 months of end of radiosurgery.

treatment modalities include pharmaceutical therapy, chemotherapy, radiotherapy, and surgical intervention ${ }^{4}$. Between 1947 and 2016, 59 cases of spinal GSD were reported in the literature. Of these 59 cases, $49 \%$ involved the cervical spine, $46 \%$ involved the thoracic spine, $17 \%$ involved the lumbar spine, and $5 \%$ involved the sacral spine ${ }^{12)}$. In almost all cases, multiple spine levels were involved. Among the 59 patients, 28 underwent surgical intervention ${ }^{12)}$. Spinal lesions can be managed by radiation therapy, vertebral osteotomy, 
braces, balloon vertebroplasty with cement augmentation, or spinal fusion with bone graft to correct instability and prevent neurologic impairment ${ }^{1,8,9)}$. Tateda et al. ${ }^{12)}$ reported that chemotherapy before surgical correction was effective. GSD patients can gain better surgical outcomes through neoadjuvant chemotherapy targeting involved tissue. In our case, neoadjuvant therapy was not administered after surgery. This is because, based on the age of the patient, it was judged that the patient's GSD state had passed the active phase. A new lesion was found 1 year later, and radiotherapy were per formed, but there is a regret that it would have been better if performed immediately after surgery. Chylothorax can occur when this disease involves the pleura or thoracic duct, and several studies report an association between GSD and chylothorax ${ }^{5,11)}$. Bilateral chylothorax is usually lethal and can cause gradual respiratory failure with an increased mortality rate. Our case showed bilateral pleural effusions 2 weeks after surgery, but the effluent was proven to be exudate, not chylothorax. Further studies are required to evaluate what causes an exudative condition.

\section{CONCLUSION}

This report described a case of GSD that involved the thoracic spine and was appropriately managed. In the present case of a middle-aged Asian woman, correction of severe thoracic instability and prevention of neurologic compromise were accomplished through PLF from C7 to T7. Recurrence was observed 1 year later, but it was appropriately treated with chemotherapy and radiotherapy.

\section{CONFLICTS OF INTEREST}

No potential conflict of interest relevant to this article was reported.

\section{REFERENCES}

1. Bruch-Gerharz D, Gerharz CD, Stege H, Krutmann J, Pohl $\mathrm{M}$, Koester R, et al.: Cutaneous lymphatic malformations in disappearing bone (Gorham-Stout) disease: a novel clue to the pathogenesis of a rare syndrome. J Am Acad Dermatol 56:S21S25, 2007

2. Gorham LW, Stout AP: Massive osteolysis (acute spontaneous absorption of bone, phantom bone, disappearing bone); its relation to hemangiomatosis. J Bone Joint Surg Am 37-a:9851004, 1955

3. Gorham LW, Wright AW, Shultz HH, Maxon FC, Jr.: Disappearing bones: a rare form of massive osteolysis; report of two cases, one with autopsy findings. Am J Med 17:674-682, 1954

4. Handl-Zeller L, Hohenberg G: Radiotherapy of Morbus GorhamStout: the biological value of low irradiation dose. $\mathrm{Br} \mathrm{J}$ Radiol 63:206-208, 1990

5. Hellyer J, Oliver-Allen H, Shafiq M, Tolani A, Druzin M, Jeng $M$, et al.: Pregnancy complicated by Gorham-Stout disease and refractory chylothorax. AJP Rep 6:e355-e358, 2016

6. Hopman SM, Van Rijn RR, Eng C, Bras J, Alders M, van der Horst CM, et al.: PTEN hamartoma tumor syndrome and Gorham-Stout phenomenon. Am J Med Genet A 158a:1719-1723, 2012

7. Kim BJ, Kim TH, Kim DJ, Noh D, Ham SJ, Lee S: A successfully treated case of gorham-stout syndrome with sternal involvement. Korean J Thorac Cardiovasc Surg 48:90-94, 2015

8. Kohno M, Aota Y, Kawai T, Murata H, Saito T: Surgical treatment of Gorham's disease with massive osteolysis of the skull and cervical spine: A case report and review of literature. NMC Case Rep J 2:80-84, 2015

9. Liu S, Zhou X, Song A, Kong X, Wang Y, Liu Y: Successful treatment of Gorham-Stout syndrome in the spine by vertebroplasty with cement augmentation: A case report and literature review. Medicine (Baltimore) 97:e11555, 2018

10. Renacci RM, Bartolotta RJ: Gorham disease: lymphangiomatosis with massive osteolysis. Clin Imaging 41:83-85, 2017

11. Takahashi A, Ogawa C, Kanazawa T, Watanabe H, Suzuki M, Suzuki N, et al.: Remission induced by interferon alfa in a patient with massive osteolysis and extension of lymphhemangiomatosis: a severe case of Gorham-Stout syndrome. J Pediatr Surg 40:E47-E50, 2005

12. Tateda S, Aizawa T, Hashimoto K, Kanno H, Ohtsu S, Itoi E, et al.: Successful management of Gorham-Stout disease in the cervical spine by combined conservative and surgical treatments: A case report. Tohoku J Exp Med 241:249-254, 2017

13. Tie ML, Poland GA, Rosenow EC, $3^{\text {rd }}$ : Chylothorax in Gorham's syndrome. A common complication of a rare disease. Chest 105:208-213, 1994 